\title{
Expression of the RNA-binding protein RBM3 is associated with a favourable prognosis and cisplatin sensitivity in epithelial ovarian cancer
}

Åsa Ehlén ${ }^{1}$, Donal J Brennan², Björn Nodin', Darran P O'Connor², Jakob Eberhard ${ }^{3}$, Maria Alvarado-Kristensson ${ }^{1}$, lan B Jeffrey ${ }^{4}$, Jonas Manjer ${ }^{5,6}$, Jenny Brändstedt ${ }^{1}$, Mathias Uhlén ${ }^{7}$, Fredrik Pontén ${ }^{8}$, Karin Jirström ${ }^{1 *}$

\begin{abstract}
Background: We recently demonstrated that increased expression of the RNA-binding protein RBM3 is associated with a favourable prognosis in breast cancer. The aim of this study was to examine the prognostic value of RBM3 mRNA and protein expression in epithelial ovarian cancer (EOC) and the cisplatin response upon RBM3 depletion in a cisplatin-sensitive ovarian cancer cell line.
\end{abstract}

Methods: RBM3 mRNA expression was analysed in tumors from a cohort of 267 EOC cases (Cohort I) and RBM3 protein expression was analysed using immunohistochemistry $(\mathrm{IHC})$ in an independent cohort of 154 prospectively collected EOC cases (Cohort II). Kaplan Meier analysis and Cox proportional hazards modelling were applied to assess the relationship between RBM3 and recurrence free survival (RFS) and overall survival (OS). Immunoblotting and $\mathrm{IHC}$ were used to examine the expression of RBM3 in a cisplatin-resistant ovarian cancer cell line A2780-Cp70 and its cisplatin-responsive parental cell line A2780. The impact of RBM3 on cisplatin response in EOC was assessed using siRNA-mediated silencing of RBM3 in A2780 cells followed by cell viability assay and cell cycle analysis.

Results: Increased RBM3 mRNA expression was associated with a prolonged RFS ( $\mathrm{HR}=0.64,95 \% \mathrm{Cl}=0.47-0.86$, $p=0.003)$ and $\mathrm{OS}(\mathrm{HR}=0.64,95 \% \mathrm{Cl}=0.44-0.95, p=0.024)$ in Cohort I. Multivariate analysis confirmed that RBM3 mRNA expression was an independent predictor of a prolonged RFS, ( $H R=0.61,95 \% \mathrm{Cl}=0.44-0.84, p=0.003)$ and OS (HR $=0.62,95 \% \mathrm{Cl}=0.41-0.95 ; p=0.028)$ in Cohort I. In Cohort II, RBM3 protein expression was associated with a prolonged $\mathrm{OS}(\mathrm{HR}=0.53,95 \% \mathrm{Cl}=0.35-0.79, p=0.002)$ confirmed by multivariate analysis $(\mathrm{HR}=0.61,95 \% \mathrm{Cl}=$ $0.40-0.92, p=0.017)$. RBM3 mRNA and protein expression levels were significantly higher in the cisplatin sensitive A2780 cell line compared to the cisplatin resistant A2780-Cp70 derivative. siRNA-mediated silencing of RBM3 expression in the A2780 cells resulted in a decreased sensitivity to cisplatin as demonstrated by increased cell viability and reduced proportion of cells arrested in the G2/M-phase.

Conclusions: These data demonstrate that RBM3 expression is associated with cisplatin sensitivity in vitro and with a good prognosis in EOC. Taken together these findings suggest that RBM3 may be a useful prognostic and treatment predictive marker in EOC.

\section{Background}

Epithelial ovarian cancer (EOC) is the leading cause of death from gynaecological malignancy and the fifth most common cause of cancer-related death in women. The poor ratio of survival to incidence in EOC is related

\footnotetext{
* Correspondence: karin.jirstrom@med.lu.se

${ }^{1}$ Center for Molecular Pathology, Department of Laboratory Medicine, Lund University, Skåne University Hospital, Malmö, Sweden

Full list of author information is available at the end of the article
}

to the high percentage of cases diagnosed at an advanced stage and the lack of effective therapies for advanced refractory disease. Despite improvements in surgical techniques and the advent of more targeted therapeutic agents, five year survival rates for EOC are only $45 \%$ [1]. Such poor statistics indicate an urgent requirement to improve on current understanding of the molecular mechanisms underlying EOC, so as to develop better early diagnostic and prognostic 
biomarkers. In addition, accurate predictive biomarkers are required to guide current treatment protocols, as well as to guide the development and application of new targeted therapies.

Since its inception over 40 years ago, the platinumbased agent cisplatin has had a major impact on cancer therapy, particularly in the treatment of testicular and ovarian cancer [2]. Standard treatment for advanced EOC involves surgical debulking followed by adjuvant chemotherapy with a combination of a platinum compound (cisplatin or carboplatin) and taxane [3]. Despite an initial response to cisplatin treatment, many patients with EOC develop resistance to the drug and relapse within a few years [4]. Cisplatin acts by forming covalent bonds with purine DNA bases which causes cross-linking of DNA and results in activation of several signal transduction pathways involved in DNA-damage repair, cell cycle arrest and apoptosis $[2,5,6]$. Several mechanisms have been implicated in cisplatin resistance, i.e. decreased drug uptake, insufficient DNA-binding of the drug, increased DNA-repair of cisplatin adducts and failure of induction of apoptosis, reviewed in $[2,5,7]$.

The RNA binding motif protein 3, RBM3, is a glycine rich protein containing a RNA-recognition motif (RRM) through which it binds to both to DNA and RNA [8]. Proteins containing specific RRMs play an important role in the stabilization of mRNA by reversibly binding to conserved sequence elements, most often AU-rich elements (AREs), in the untranslated regions (UTRs) of the mRNA resulting in either stabilization or destabilization of the mRNA [9]. The RBM proteins, 10 of which have been described, contain between one and four copies of the RRM consensus sequence [10]. The RRM domain is evolutionary conserved across species and found in virtually every cellular organelle in which RNA is present suggesting an important but as yet not fully understood functionality [10]. RBM3, initially identified in a fetal brain cDNA library [11] is one of three Xchromosome related RBM-genes (RBMX, RBM3, RBM10) mapped to Xp11.23 [12] and is expressed in various human fetal tissues as well as being one of the earliest proteins induced by hypothermia [13]. Following an antibody-based proteomics biomarker discovery strategy using the Human Protein Atlas (HPA) (http://www. proteinatlas.org) $[14,15]$ we recently demonstrated an association between nuclear RBM3 expression in breast cancer and a significantly improved survival, particularly in estrogen receptor (ER) positive tumors [16].

In the present study, the prognostic value of RBM3 was examined in two independent EOC cohorts, both at the mRNA levels (Cohort I) and protein levels (Cohort II), whereby RBM3 was found to be associated with a good prognosis in both cohorts. RBM3 expression was also examined in vitro using the cisplatin sensitive ovarian cancer cell line A2780 and its cisplatin resistant derivative A2780-Cp70. The relationship between RBM3 expression and cisplatin response in vitro was examined using small interfering RNA (siRNA) mediated RBM3 knockdown in the A2780 cells which resulted in a decreased sensitivity to cisplatin as demonstrated by an increased cell viability and reduced proportion of cells G2/M-phase arrest following cisplatin treatment.

\section{Methods \\ Patients \\ Cohort I}

Cohort I comprised of 285 cases of serous and endometroid carcinoma of the ovary, fallopian tube and peritoneum. The cohort has been described previously [17]. The majority of patients underwent laparotomy for staging and debulking and subsequently received first-line platinum/taxane based chemotherapy. In most cases, tumor tissue was excised at the time of primary surgery, prior to the administration of chemotherapy. Eighteen patients who had received neoadjuvant platinum based chemotherapy were also included in the cohort but excluded from this study hence the total number or patients examined was 267. Optimal debulking was defined as less than $1 \mathrm{~cm}$ (diameter) residual disease, and sub-optimal debulking was more than $1 \mathrm{~cm}$ (diameter) residual disease. Recurrence-free survival (RFS) was defined as the time interval between the date of diagnosis and the first confirmed sign of disease recurrence based on GCIG definitions. Overall survival (OS) was defined as the time interval between the date of histological diagnosis and the date of death from any cause. Median follow up was 29 months (range 0-214 months).

RNA was extracted from tumors and hybridized to Affymetrix U133 Plus 2 arrays as previously described [17]. Complete expression data were downloaded from GEO (http://www.ncbi.nlm.nih.gov/geo) (accession GSE9899). R package "Affy" (http://www.bioconductor. org) was used to normalize the CEL files using the RMA method [18]. For RBM3 analysis normalized gene expression values were extracted from the dataset and used without modification. Tumor samples were classified using a previously published method [19].

\section{Cohort II}

This cohort is a merge of all incident cases of epithelial ovarian cancers in the large, population-based prospective cohort studies Malmö Diet and Cancer Study [20] $(\mathrm{n}=101)$ and Malmö Preventive Medicine Study [21] $(\mathrm{n}=108)$ until Dec $31^{\text {st }} 2008$. Thirty-five patients participated in both studies, and archival tumor tissue could be retrieved from 154 of the total number of 174 cases. After a median follow-up of 2.65 years (range 0-21), 105 patients $(68.2 \%)$ were dead and 49 $(31.8 \%)$ alive. 
All tumors were re-evaluated regarding histological subtype and histological grade. Information regarding clinical stage was obtained from the medical charts, following the standardized FIGO classification of tumor staging. Information on residual tumor after surgery was not available. Standard adjuvant therapy was platinumbased chemotherapy, from the 1990s given in combination with paclitaxel.

\section{Tissue microarray construction}

Prior to TMA-construction, all cases were histopathologically re-evaluated on haematoxylin and eosin stained slides. Areas representative of cancer were then marked and TMAs constructed as previously described [22]. In brief, 2-4 $1.0 \mathrm{~mm}$ cores were taken from each tumor and mounted in a new recipient block using a semiautomated arraying device (TMArrayer; Pathology Devices, Inc, Westminster, MD, USA).

\section{RBM3 antibody generation and immunohistochemistry}

PrEST $[23,24]$ antigen was injected subcutaneously into BALB/c mice (4-6 weeks old, female) at three weeks intervals. The antigen was mixed with complete Freund's adjuvant for the first injection and incomplete Freund's adjuvant for the following injections. Three days before infusion, the mouse was last challenged with antigen intravenously. Hybridomas were generated by fusion of mouse splenocytes with the Sp2/0 myeloma cell line. Cell lines that showed positive results in ELISA, Western blot (WB) and immunohistochemistry (IHC) were selected for subcloning.

For immunohistochemical analysis of RBM3 in Cohort II, $4 \mu \mathrm{m}$ TMA-sections were automatically pretreated using the PT-link system (DAKO, Copenhagen, Denmark) and then stained in a Techmate 500 (DAKO, Copenhagen, Denmark) with the mouse monoclonal anti-RBM3 antibody (AAb030038, Atlas Antibodies AB, Stockholm, Sweden) diluted 1:5000. Estrogen receptor (ER) and progesterone receptor (PR) expression were assessed following, heat-mediated antigen retrieval which was performed using microwave treatment for $2 \times 5 \mathrm{~min}$ in a citrate buffer before being processed in the Ventana Benchmark system (Ventana Medical Systems Inc, AZ) using pre-diluted antibodies to ER (Anti-ER, clone 6F11) and PR (Anti-PgR, clone 16).

\section{Analysis of immunohistochemical staining}

For assessment of nuclear RBM3 expression, both the fraction of positive cells and staining intensity were taken into account using a modification of the previously applied semiquantitative scoring system [16]. Nuclear fraction (NF) was categorized into four groups, namely $0(0-1 \%), 1$ (2-25\%), $2(26-75)$ and $3(>75 \%)$ and nuclear staining intensity (NI) denoted as $0-2$, whereby $0=$ negative, $1=$ intermediate and $2=$ moder ate-strong intensity. A combined nuclear score (NS) of NFxNI, which had a range of 0 to 6 , was then constructed. Cytoplasmic staining intensity was denoted as $0=$ negative, $1=$ mild and $2=$ moderate-strong, and the fraction of positive cells not taken into account. ER and PR negativity was defined as $<10 \%$ positively staining nuclei.

\section{Cell lines and reagents}

The human ovarian cancer cell line A2780 and the cisplatin-resistant variant A2780-Cp70 (received as a gift from Prof R Brown, Imperial College, London) were maintained in RPMI-1640 supplemented with glutamine, $10 \%$ fetal bovine serum and $1 \%$ pencillin/streptomycin in a humidified incubator of $5 \% \mathrm{CO} 2$ at $37^{\circ} \mathrm{C}$. Cisplatin (Sigma-Aldrich, St. Louis, MO, USA) was dissolved in $0.9 \% \mathrm{NaCl}$ to a stock solution of $1 \mathrm{mg} / \mathrm{ml}$ and added to cells to the final concentration $(1-100 \mu \mathrm{M})$.

\section{Real-time quantitative PCR and Western Blotting}

Total RNA isolation (RNeasy, QIAgen, Hilden, Germany), cDNA synthesis (Reverse Transcriptase kit, Applied Biosystems, Warrington, UK) and quantitative real-time PCR (qRT-PCR) analysis with SYBR Green PCR master mix (Applied Biosystems) were performed as described [25,26]. Quantification of expression levels were calculated by using the comparative $\mathrm{Ct}$ method, normalization according to house keeping genes; HMBS (forward primer: 5'-GGC AAT GCG GCT GCA A-3', reverse primer: 5'-GGG TAC CCA CGC GAA TCA C3'), SDHA (forward primer: 5'-TGG GAA CAA GAG GGC ATC TG-3', reverse primer 5'-CCA CCA CTG CAT CAA ATT CAT G-3') and UBC (forward primer: 5'-ATT TGG GTC GCG GTT CTT G-3', reverse primer: 5'-TGC CTT GAC ATT CTC GAT GGT-3'). For RBM3 amplification, forward primer with sequence $5^{\prime}$ CTT CAG CAG TTT CGG ACC TA-3' and reverse primer with sequence $5^{\prime}$-ACC ATC CAG AGA CTC TCC GT-3' were used. All primers were designed using Primer Express (Applied Biosystems).

For immunoblotting, cells were lysed in ice-cold lysis buffer $(150 \mathrm{mM} \mathrm{NaCl}, 50 \mathrm{mM}$ Tris-HCL pH 7.5, 1\% Triton X-100, $50 \mathrm{mM} \mathrm{NaF}, 1 \mathrm{mM}$ Na3VO4, $1 \mathrm{mM}$ phenylmethylsulfonyl fluoride (PMSF)) and supplemented with protease inhibitor cocktail Complete Mini (Roche, Basel, Switzerland). For Western blotting, 20-50 $\mu \mathrm{g}$ of protein were separated on 15\% SDS-PAGE gels and transferred onto nitrocellulose membranes (Hybond ECL, Amersham Pharmacia Biotech, Buckinghamshire, UK). The membranes were probed with primary antibodies followed by horseradish peroxidase (HRP)-conjugated secondary antibodies (Amersham Life Science, Alesbury, U.K.) and visualized using the Enhanced 
ChemiLuminescence detection system (ECL) and ECL films (Amersham Pharmacia Biotech). RBM3 was detected by the mouse monoclonal anti-RBM3 antibody (AAb030038, Atlas Antibodies AB, Stockholm, Sweden) diluted 1:500 in blocking solution (5\% BSA, 1× PBS, $0.1 \%$ Tween20), Bax by a polyclonal antibody (BD Pharmingen, San Diego, CA, USA) diluted 1:1000 and Bcl-2 using a monoclonal antibody diluted 1:250 (Santa Cruz, Biotechnology, Santa Cruz, CA, USA). Membranes were stripped and re-probed with an anti- $\beta$-actin antibody (Santa Cruz, Biotechnology, Santa Cruz, CA, USA) at a dilution of 1:1000, to provide a loading control.

\section{Cell pellet arrays}

Cell lines were fixed in $4 \%$ formalin and processed in gradient alcohols. Cell pellets were cleared in xylene and washed multiple times in molten paraffin. Once processed, cell lines were arrayed in duplicate $1.0 \mathrm{~mm}$ cores using a manual tissue arrayer (Beecher Inc, WI) and IHC was performed on $4 \mu \mathrm{m}$ sections using the RBM3 1B5 antibody diluted 1:1000.

\section{siRNA knockdown of RBM3 gene expression}

Transfection with siRNA against RBM3 (Applied Biosystems, Carlsbad, Ca) or control siRNA (Applied Biosystems) was performed with Lipofectamine 2000 (Invitrogen, Carlsbad, CA) with a final concentration of $50 \mathrm{nM}$ siRNA. All siRNA experiments were performed using three independent RNA oligonucleotides (\#58, \#59 and \#60) targeting RBM3.

\section{WST-1 cell viability assay}

The effect of cisplatin on cell viability was determined by the WST-1 assay (Roche Applied Science, Mannheim, Germany) according to the manufacturer's recommendation. A2780 and A2780-Cp70 cells were seeded in 96-well plates at the density of 2500 cells/well in $100 \mu \mathrm{l}$ appropriate medium a day before addition of cisplatin. Cells were treated with cisplatin $(0-100 \mu \mathrm{M})$ for $1 \mathrm{~h}$ followed by 24, 48 or $72 \mathrm{hrs}$ recovery in fresh drug-free media. Samples were made in triplicate. Ten microliters WST-1 solution was added per well and incubated at $37^{\circ} \mathrm{C}$ for $4 \mathrm{hrs}$. The absorbance of each well was measured using a scanning multiwell spectrophotometer, ELISA reader, at the wavelength of $450 \mathrm{~nm}$ and reference wavelength of $690 \mathrm{~nm}$.

\section{Flow cytometry}

For cell cycle phase analysis, cells were fixed in 70\% ethanol for 30 minutes at $-20^{\circ} \mathrm{C}$ followed with washing with PBS and centrifugation. In order to label DNA, pellets were resuspended in Vindelöv solution $(3.5 \mu \mathrm{M}$ Tris- $\mathrm{HCl} \mathrm{pH}$ 7.6, $10 \mathrm{mM} \mathrm{NaCl}, 10 \mu \mathrm{g} / \mathrm{ml}$ propidium iodide, $20 \mathrm{ug} / \mathrm{ml}$ RNase and $0.1 \% \mathrm{v} / \mathrm{v} \mathrm{NP40)}$ and incubated in the dark for 20 minutes on ice. The cell cycle analysis was performed by flow cytometry analysis using FACS Calibur (BD Biosciencies, San José, CA), counting in total $1 \times 10^{4}$ cells. Gating of G0/G1-, Sand G2/M-populations was performed manually using the FlowJo software (version 6.4.7, Tree Star, Inc. Ashland, OR).

The fraction of apoptotic and necrotic cells were analyzed by flow cytometry using AnnexinV-APC and 7AAD (BD Pharmingen, San Diego, CA) staining according to manufacturer's instructions. Briefly, cells were harvested by trypsinization, washed twice in cold PBS and resuspended in $1 \times$ Annexin V Binding buffer (BD Pharmingen, San Diego, CA). Cells were stained with AnnexinV-APC antibody and 7AAD and subjected to flow cytometric analysis using a FACSCalibur flow cytometry (BD Biosciencies, San José, CA) to determine the percentage of AnnexinV and 7AAD positive cells. The results are given as the mean of three independent experiments, bars indicate standard error of mean.

\section{Statistics}

Spearman's Rho, Chi-square and Kruskal-Wallis tests were used for comparison of RBM3 expression and relevant clinicopathological characteristics. Kaplan-Meier analysis and log rank test were used to illustrate differences in recurrence free survival (RFS) and overall survival (OS) according to RBM3 expression. Cox regression proportional hazards models were used to estimate the impact of RBM3 expression on RFS and OS in both uni- and multivariate analysis, adjusted for stage and differentiation grade (both cohorts) and volume of residual tumor ( 0 vs $>0)$ in Cohort I. Patients who had received neoadjuvant chemotherapy in Cohort I $(n=18)$ were excluded from the survival analyses. All calculations were performed using SPSS version 15.0 (SPSS Inc, Chicago, IL). All statistical tests were two-sided and a $\mathrm{p}$ value $<0.05$ was considered statistically significant. The experimental data are expressed as mean \pm SEM of at least three independent experiments. Statistical significance of differences between means was determined by one-way ANOVA followed by Duncan's multiple range test or Student's $t$-test.

\section{Results \\ Validation of the RBM3 antibody}

The specificity of the RBM3 antibody was confirmed by siRNA-mediated knockdown of RBM3 in A2780 cells. IHC performed on formalin fixed, paraffin embedded siRNA transfected A2780 cells revealed a marked decrease in immunoreactivity in the RBM3 knockdown cells compared to controls as visualized by IHC on cell pellets (Fig. 1A) and Western blotting (Fig. 1B). 


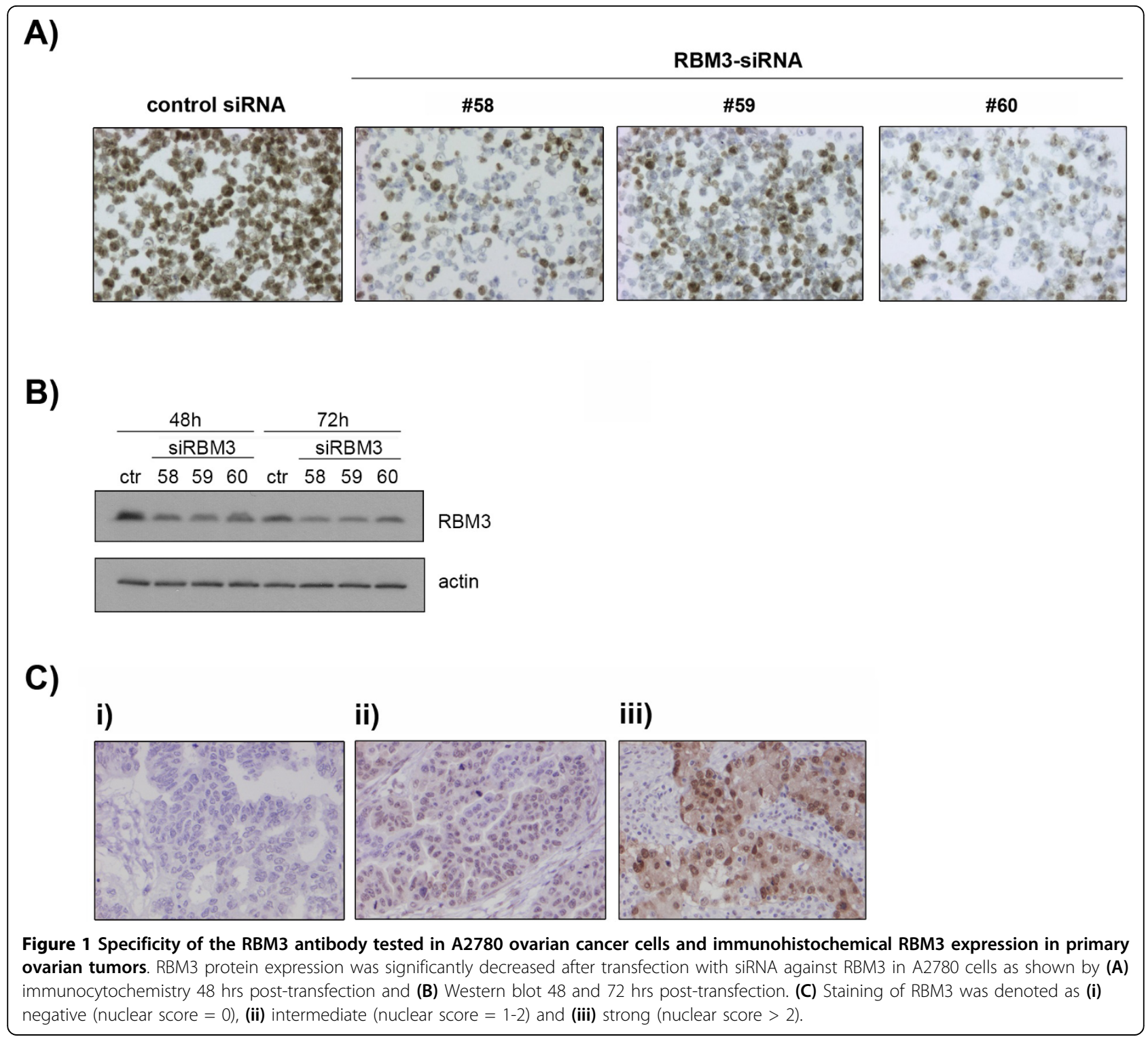

RBM3 expression and association to clinicopathological characteristics in epithelial ovarian cancer

Having previously demonstrated that RBM3 was associated with a less aggressive breast cancer phenotype [16] we sought to examine the relationship between RBM3 mRNA and protein expression and clinicopathological characteristics in two independent EOC cohorts. In Cohort I, increased RBM3 mRNA levels were not associated with any clinicopathological characteristics (Table 1).

In Cohort II, following antibody optimisation and staining, it was possible to evaluate the expression of RBM3 protein in 151 cases (98\%). Images representing different patterns of expression are shown in Figure 1C. Using the combined score, 57 (38\%) tumors lacked RBM3 nuclear RBM3 staining, and 94 (63\%) tumors expressed RBM3 in various intensities and fractions. For statistical purposes, tumors were grouped into negative $=0$ (combined NS $0-1)$, intermediate $=1$ (combined NS 2-3) and strong $=2$ (combined NS > 3). As visualized in Table 1, RBM3 NS was not associated with histological subtype, disease stage or differentiation grade. Cytoplasmic staining was only present in 27 (18\%) cases, and therefore not accounted for in the statistics. There was no significant association between RBM3 and ER or PR expression (data not shown).

Increased RBM3 mRNA levels and protein expression are associated with a prolonged survival in ovarian cancer patients

We proceeded to investigate the relationship between RBM3 expression and clinical outcome. In Cohort I, 
Table 1 Correlations between clinicopathological characteristics and RBM3 mRNA (Cohort I) levels and protein expression (Cohort II)

\begin{tabular}{|c|c|c|c|c|c|c|c|}
\hline \multirow[b]{2}{*}{ RBM3 score } & \multicolumn{2}{|c|}{ Cohort I } & & \multicolumn{2}{|c|}{ Cohort II } & \multirow[b]{2}{*}{2} & \multirow[b]{3}{*}{ p-value } \\
\hline & low & high & & 0 & 1 & & \\
\hline n (\% for columns) & $120(45.6)$ & $143(54.4)$ & $p$-value & $74(49.0)$ & $50(33.1)$ & $27(17.9)$ & \\
\hline \multicolumn{8}{|l|}{ Histological subtype } \\
\hline mucinuos & $0(0.0)$ & $0(0.0)$ & $0.887^{\dagger}$ & $4(5.4)$ & $4(8.0)$ & $3(11.1)$ & $0.395^{\dagger}$ \\
\hline serous & $111(92.5)$ & $133(93.0)$ & & $42(56.8)$ & $31(62.0)$ & $16(59.3)$ & \\
\hline endometroid & $9(7.5)$ & $9(6.3)$ & & $17(23.0)$ & $12(24.0)$ & $5(18.5)$ & \\
\hline clear cell & $0(0.0)$ & $0(0.0)$ & & $6(8.1)$ & $1(2.0)$ & $2(7.4)$ & \\
\hline Brenner & $0(0.0)$ & $0(0.0)$ & & $0(0.0)$ & $0(0.0)$ & $1(3.7)$ & \\
\hline adenocarcinoma nos & $0(0.0)$ & $1(0.7)$ & & $5(6.8)$ & $2(4.0)$ & $0(0.0)$ & \\
\hline \multicolumn{8}{|l|}{ Differentiation grade } \\
\hline high & $3(2.5)$ & $14(9.8)$ & 0.079 & $2(2.7)$ & $2(4.0)$ & $3(11.1)$ & 0.084 \\
\hline intermediate & $40(33.3)$ & 48 (33.6) & & $17(23.0)$ & $13(26.0)$ & 9 (33.3) & \\
\hline low & $77(64.2)$ & $78(54.5)$ & & $55(74.3)$ & $35(70.0)$ & 15 (55.6) & \\
\hline missing & $0(0.0)$ & $3(2.1)$ & & $0(0.0)$ & $0(0.0)$ & $0(0.0)$ & \\
\hline \multicolumn{8}{|l|}{ Stage } \\
\hline I & $9(7.5)$ & $15(10.5)$ & 0.070 & $14(18.9)$ & $7(14.0)$ & $5(18.5)$ & 0.760 \\
\hline$\|$ & $3(2.5)$ & $15(10.5)$ & & $5(6.8)$ & $9(18.0)$ & $3(11.1)$ & \\
\hline III & $101(84.2)$ & $104(72.7)$ & & $40(54.1)$ & $26(52.0)$ & 8 (29.6) & \\
\hline IV & $7(5.8)$ & $9(6.3)$ & & 9 (12.2) & $6(12.0)$ & 7 (25.9) & \\
\hline missing & $0(0.0)$ & $0(0.0)$ & & $6(8.1)$ & $2(4.0)$ & $4(14.8)$ & \\
\hline
\end{tabular}

Correlations were calculated using Spearman correlation test unless other specified.

${ }^{\dagger}$ Kruskal-Wallis test (two-sided).

Kaplan Meier analysis demonstrated that increased RBM3 mRNA levels were associated with a significantly prolonged RFS and OS (Fig. 2A and 2B). Cox univariate analysis confirmed this association with an improved RFS (HR $=0.64,95 \% \mathrm{CI}=0.47-0.86, p=0.003)$ and $\mathrm{OS}$ $(\mathrm{HR}=0.64,95 \% \mathrm{CI}=0.44-0.95, p=0.024)($ Table 2$)$. Multivariate analysis controlling for age, disease stage, differentiation grade and residual tumor volume (Table 2) confirmed that RBM3 expression was an independent predictor of RFS (HR $=0.61,95 \% \mathrm{CI}=0.44-0.84, p=$ $0.003)$ and $\mathrm{OS}(\mathrm{HR}=0.62,95 \% \mathrm{CI}=0.41-0.95 ; p=$ $0.028)$. The independent beneficial prognostic value of RBM3 expression for both RFS and OS was retained when subset analysis of tumours of pure ovarian origin ( $\mathrm{n}=243$ ) was performed (data not shown).

In Cohort II, Kaplan Meier analysis revealed a stepwise association between RBM3 expression and OS whereby RBM3 positive tumors had a significantly improved OS compared to RBM3 negative tumors (Fig. $2 \mathrm{C}$ ), justifying a dichotomisation into negative versus any expression (Fig. 2D). Cox regression analysis showed a significantly improved OS for RBM3 positive tumors in both univariate $(\mathrm{HR}=0.53,95 \% \mathrm{CI}=0.35-0.79, p=$ 0.002), and multivariate analysis $(\mathrm{HR}=0.61,95 \% \mathrm{CI}=$
$0.40-0.92, p=0.017)$, adjusted for age, stage and differentiation grade (Table 2).

RBM3 levels are higher in cisplatin-sensitive than cisplatin-resistant ovarian cancer cells

RBM3 protein expression, assessed by both Western blotting and IHC, was higher in the parental A2780 cells compared to their cisplatin-resistant derivative A2780-Cp70 (Fig. 3A and 3B). Real-time quantitative PCR (qRT-PCR) confirmed a similar difference whereby there was a three fold higher level of RBM3 mRNA in the A2780 compared to the A2780-Cp70 cell line (Fig. 3C). As RBM3 has been found to be associated with proliferation $[27,28]$, we compared the growth rate of the two cell lines, and were unable to demonstrate any significant differences in proliferation (Fig. 3D).

Cell viability assay confirmed cisplatin resistance in the A2780-Cp70 cells relative to the A2780 cells $(p<$ 0.05 ; Fig. 3E). The proportion of cells arrested in G2/M (Fig. 3F) and apoptotic/necrotic cells (Fig. 3G) following cisplatin treatment was also significantly reduced in the A2780-Cp70 cells. Notably, there was a significant difference in cell cycle phase distribution between untreated A2780 and A2780-Cp70 cells (Fig. 3F) with a 


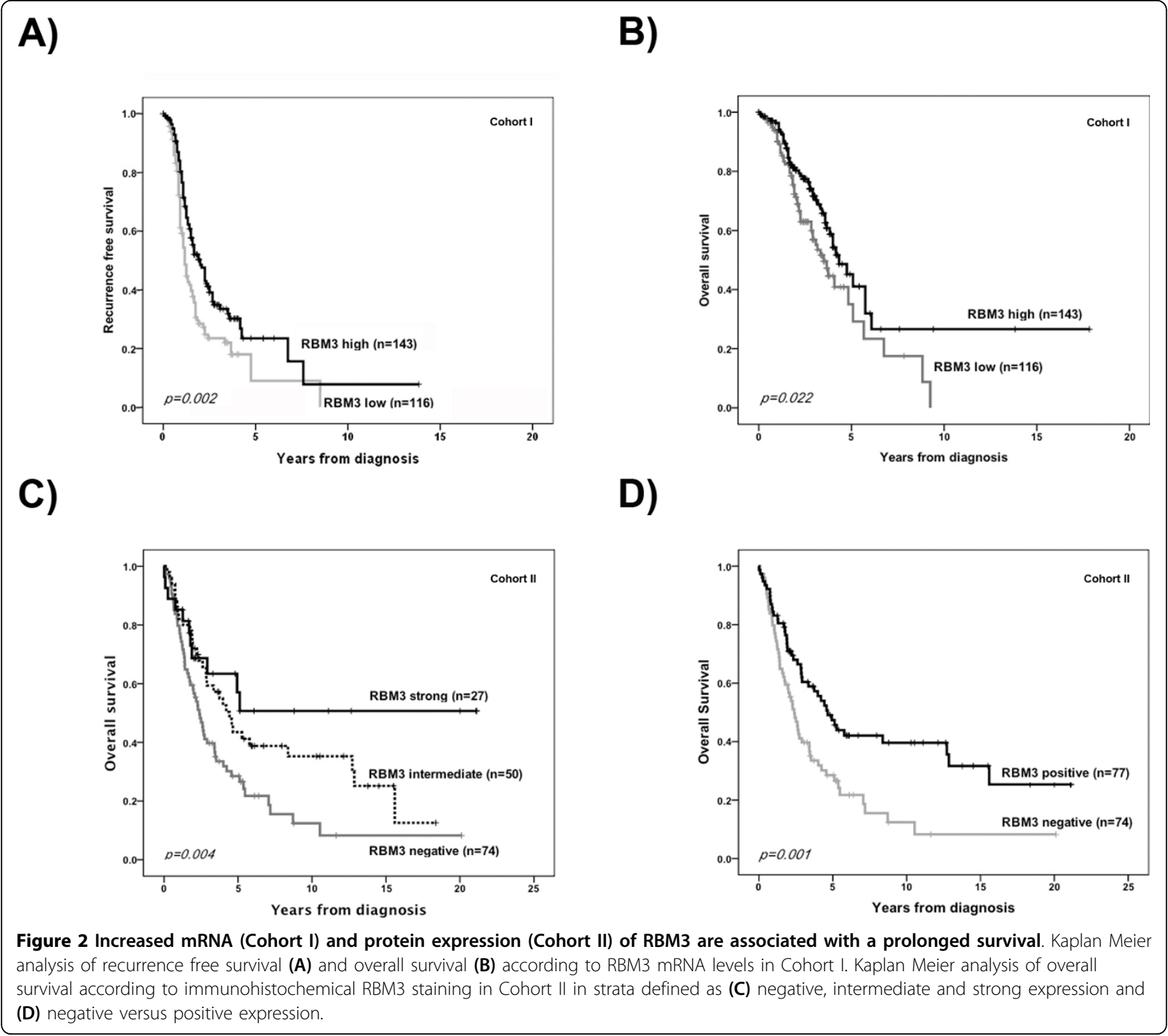

larger proportion of cells in G2/M in the A2780-Cp70 cells, but no significant difference in apoptosis (Fig. 3G) was observed. However, there was a trend towards a higher fraction of apoptotic cells in untreated A2780Cp70 cells.

\section{Downregulation of RBM3 significantly reduces cisplatin sensitivity in ovarian cancer cells}

The relationship between RBM3 and cisplatin response was then examined using siRNA mediated gene silencing, whereby the cisplatin-sensitive A2780 cells were transfected with three different RBM3-specific siRNA:s or scrambled control siRNA prior to cisplatin treatment. Subsequent determination of cisplatin response as measured by cell cytotoxicity assay and cell cycle analysis revealed that RBM3 knockdown in A2780 cells significantly decreased their sensitivity to cisplatin (Fig. 4A). This effect was evident for three independent RBM3-specific siRNAs. Furthermore, cisplatin induced G2/M arrest was significantly less pronounced in siRBM3 transfected A2780 cells compared to si-control transfected cells (Fig. 4B). There was also a decreased, however non-significant, percentage of apoptotic cells in siRBM3 transfected cells compared to controls (Fig. 4C).

Given the previously demonstrated relationship between RBM3 and apoptosis-regulating proteins [29], we also compared the levels of $\mathrm{Bcl}-2$ and Bax in siRBM3 transfected cells and controls. In line with previous findings [30], Bcl-2 could barely be detected in the A2780 cells while elevated levels were observed in A2780-Cp70 cells. (Additional file 1A). In A2780-Cp70 cells, Bax levels were lower than in A2780 cells, but Bax levels 
Table 2 Cox uni- and multivariate analysis of recurrence free and overall survival according to RBM3 mRNA levels (Cohort I) and protein expression (Cohort II)

\begin{tabular}{|c|c|c|c|c|c|c|c|c|}
\hline & \multicolumn{2}{|c|}{ Recurrence free survival } & \multicolumn{6}{|c|}{ Overall survival } \\
\hline & & HR $(95 \% \mathrm{Cl})$ & & $p$-value & & $\mathrm{HR}(95 \% \mathrm{Cl})$ & & $p$-value \\
\hline \multicolumn{9}{|l|}{ Cohort I } \\
\hline & & & Univariate & & & & Univariate & \\
\hline low & $\left(n=115, n_{\text {event }}=85\right)$ & 1.00 & & & $\left(n=115, n_{\text {event }}=54\right)$ & 1.00 & & \\
\hline \multirow[t]{2}{*}{ high } & $\left(n=142, n_{\text {event }}=89\right)$ & $0.64(0.47-0.86)$ & & 0.003 & $\left(n=139, n_{\text {event }}=50\right)$ & $0.64(0.44-0.95)$ & & 0.024 \\
\hline & & & Multivariate & & & & Multivariate & \\
\hline low & $\left(n=103, n_{\text {event }}=77\right)$ & 1.00 & & & $\left(n=103, n_{\text {event }}=51\right)$ & 1.00 & & \\
\hline high & $\left(\mathrm{n}=119, \mathrm{n}_{\text {even }} \mathrm{t}=71\right)$ & $0.61(0.44-0.84)$ & & 0.003 & $\left(n=117, n_{\text {even }} t=50\right)$ & $0.62(0.41-0.95)$ & & 0.028 \\
\hline \multicolumn{9}{|l|}{ Cohort II } \\
\hline & & & & & & & Univariate & \\
\hline negative & $x$ & $x$ & $x$ & $x$ & $\left(n=74, n_{\text {event }}=33\right)$ & 1.00 & & \\
\hline \multirow[t]{2}{*}{ positive } & $x$ & $x$ & $x$ & $x$ & $\left(n=77, n_{\text {event }}=44\right)$ & $0.53(0.35-0.79)$ & & 0.002 \\
\hline & & & & & & & Multivariate & \\
\hline negative & $x$ & $x$ & $x$ & $x$ & $\left(n=68, n_{\text {event }}=29\right)$ & 1.00 & & \\
\hline positive & $x$ & $x$ & $x$ & $x$ & $\left(n=71, n_{\text {event }}=42\right)$ & $0.61(0.40-0.92)$ & & 0.017 \\
\hline
\end{tabular}

Multivariate analysis included adjustment for age (continuous) stage (I-II vs III-IV), grade (I-II vs III) and residual disease (none vs any, only available for Cohort I).

were not considerably altered by down-regulation of RBM3 (Additional file 1B-C).

Cisplatin treatment did not affect RBM3 protein expression or the siRNA mediated down-regulation of RBM3 (Additional file 2). The effects of RBM3 downregulation in A2780 cells in the absence of cisplatin were also investigated and, in agreement with previous studies [27], siRBM3 transfected A2780 cells showed a significantly reduced cell viability, a slightly higher proportion of cells in $\mathrm{G} 2 / \mathrm{M}$ and no effect on apoptosis (Additional file 3).

\section{Discussion}

This investigation of the prognostic value of RBM3 in EOC reveals that RBM3 is an independent prognostic marker at both mRNA and protein levels. Gene expression analysis in a cohort of 267 EOC cases showed that high RBM3 mRNA expression was an independent predictor of a significantly improved RFS and OS. Immunohistochemical analysis in an independent cohort of 154 EOC cases demonstrated that RBM3 protein expression was associated with a significantly improved OS in univariate and multivariate analysis. This is in line with previous findings from two independent breast cancer cohorts where RBM3 was associated with more favourable clinicopathological parameters and a significantly improved survival, irrespective of adjuvant treatment [16]. However, since platinum-based chemotherapy is a fundamental aspect of current EOC treatment regimens, we hypothesized that RBM3 might enhance platinumsensitivity in vitro. We initially confirmed lower RBM3 protein levels in the cisplatin-resistant ovarian cancer cell line A2780-Cp70 compared to their parental cisplatin-sensitive A2780 cells and using RNAi techniques, we demonstrated that silencing of RBM3 led to a decreased cisplatin response in ovarian cancer cells.

Taken together, these data demonstrate that RBM3 is a marker of good prognosis in EOC and a predictor of response to platinum-based chemotherapy, most likely a combination of both, particularly in the light of the previously demonstrated good prognosis associated with RBM3 expression in breast cancer patients, where the vast majority of patients received no adjuvant systemic chemotherapy [16]. While future in-depth studies are warranted to further elucidate the functional mechanisms underlying RBM3's role in cisplatin-mediated cell death, the in vitro data presented here provide sufficient evidence to support the hypothesis that, in addition to being a beneficial prognostic biomarker, RBM3 might also predict cisplatin response in EOC. Further studies are required to evaluate the role of RBM3 in predicting response to other platinum based agents, particularly in the setting of a prospective randomised control trial whereby stratification according to different treatment regimens can be performed.

Some aspects on the results presented here merit further attention. The reduced cytotoxic effect of cisplatin in siRBM3 transfected cells was to a large extent reflected by cell cycle alterations, e.g. a lower percentage of cells arrested in $\mathrm{G} 2 / \mathrm{M}$ phase rather than by a decreased percentage of apoptotic cells. Cisplatin treatment is known to induce both cell cycle arrest and 
A)

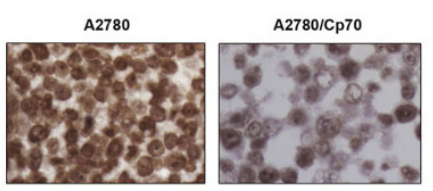

D)

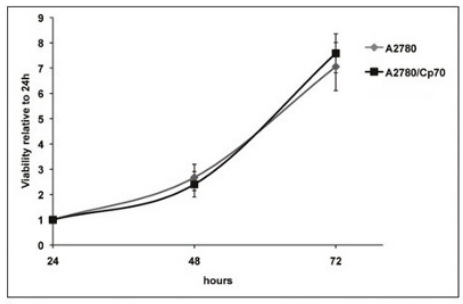

B)

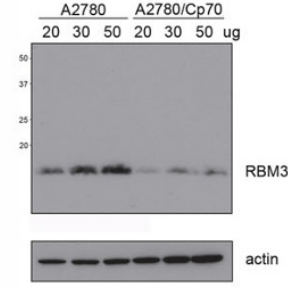

E)

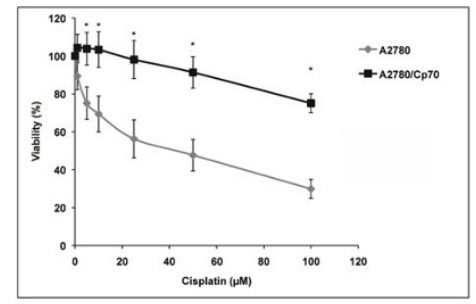

C)

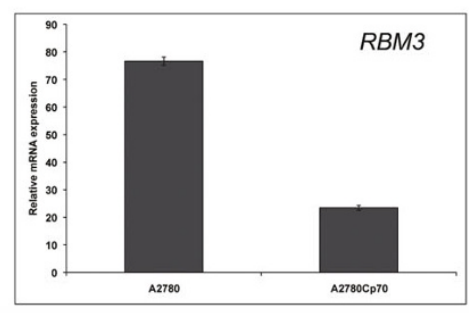

F)

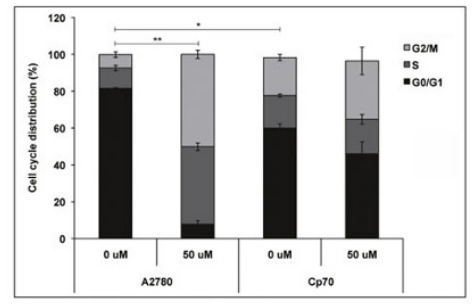

G)

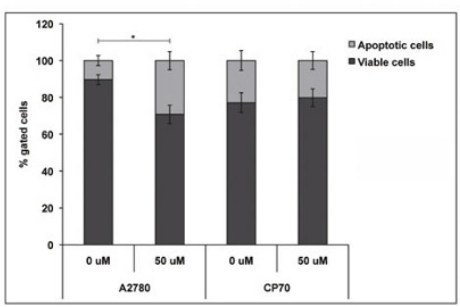

Figure 3 Expression of RBM3 in the cisplatin-sensitive A2780 ovarian cancer cell line compared to the cisplatin-resistant cell line A2780-Cp70 and the effects of cisplatin treatment on viability, cell cycle phase distribution and apoptosis in A2780 and A2780-Cp70 cell lines. (A) Immunocytochemical staining and (B) immunoblotting of RBM3 showing substantially lower RBM3 protein expression in the cisplatin-resistant A2780-Cp70 cell line compared to its parental cisplatin-sensitive A2780 cell line. (C) Relative mRNA expression was also reduced in the A2780-Cp70 cells compared to A2780 cells as shown by qRT-PCR analysis. Data shown are mean \pm SEM of a representative experiment of two independent experiments performed in triplicate. (D) Cell growth illustrated as viability ratio 48:24 and 72:24 hrs measured by WST-1 demonstrating no difference between A2780 and A2780-Cp70 cells. Data shown are mean \pm SEM of a representative experiment of three independent experiments performed in triplicate (E) Cell viability was evaluated by WST-1 assay in A2780 and A2780-Cp70 cells treated with cisplatin $(1,5,10,25,50$ and $100 \mu \mathrm{M})$ for $1 \mathrm{~h}$ followed by $48 \mathrm{hrs}$ culture in fresh drug-free media. Data are presented as mean values from four independent experiments performed in triplicates presented as percentage of viable cells as compared with untreated cells. Error bars represent SEM. (F-G) A2780 and A2780-Cp70 cells were treated with $50 \mu \mathrm{M}$ cisplatin for $1 \mathrm{~h}$ followed by 48 hrs culture in fresh drug-free media followed by flow cytometric analysis of $(\mathbf{F})$ cell cycle phase distribution and $(\mathbf{G})$ fraction of apoptotic cells. Data are presented as mean value from three independent experiments presented as fold change of cisplatin treatment. Error bars represent SEM.

apoptosis (reviewed in [7]), which was confirmed here in A2780 cells. However, while cisplatin treatment resulted in a significantly decreased cell cycle arrest in siRBM3 transfected A2780 cells, the percentage of apoptotic cells was not significantly reduced. Notably, despite being one of the most cisplatin sensitive ovarian cancer cell lines, A2780 cells have been shown to have a relatively low percentage of apoptotic cells following cisplatin treatment [30]. As there was no evident difference in cell cycle characteristics between siRBM3-transfected and control-transfected A2780 cells without cisplatin treatment, but yet a significantly reduced proportion of siRBM3 treated cells in G2/M-phase arrest following cisplatin treatment, the main effects of RBM3 on cisplatin sensitivity might be reflected in cell cycle distribution rather than apoptosis. Although RBM3 mRNA expression has previously been associated with the pro-apoptotic Bax gene in breast cancer [29], we were unable to demonstrate a down-regulation of Bax protein in siRBM3 transfected A2780 cells, further supporting the theory that RBM3 promotes cisplatin sensitivity through cell-cycle regulation.

It is evident that RBM3 is up-regulated in response to various conditions causing cellular stress, i.e. hypothermia [13,31,32] hypoxia [26], serum starvation [28] and exposure to microgravity [33]. We did not see an 
A)

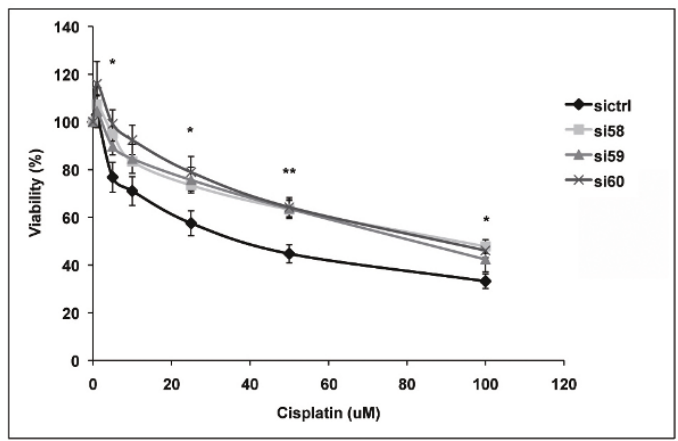

B)

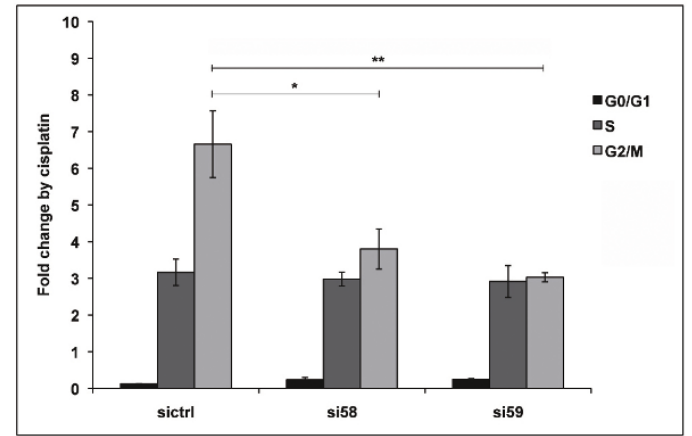

C)

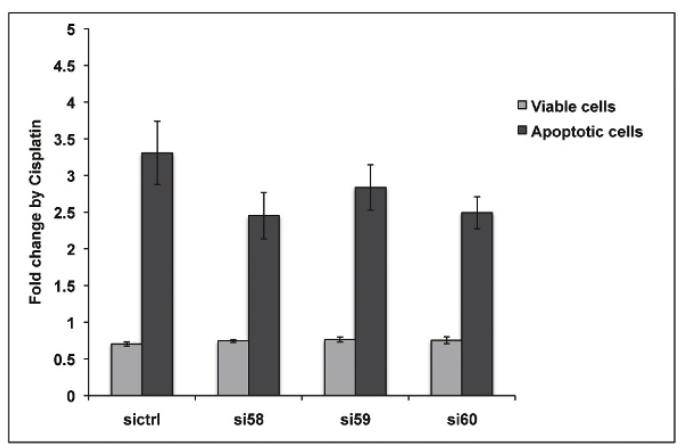

Figure 4 Down-regulation of RBM3 significantly reduces cisplatin sensitivity in ovarian cancer cells. siRBM3 transfected A2780 cells were, 24 hrs post-transfection, treated with various concentrations of cisplatin for $1 \mathrm{~h}$ followed by $48 \mathrm{hrs}$ culture in fresh drug-free media. (A) Cell viability was evaluated by WST-1 assay in siRBM3 transfected A2780 cells treated with cisplatin (1, 5, 10, 25, 50 and $100 \mu M)$. Data are presented as mean values from six independent experiments performed in triplicates presented as percentage of viable cells as compared with untreated cells. Error bars represent SEM. (B) Cell cycle phase distribution and (C) fraction of apoptotic cells were analysed by flow cytometry in siRBM3 transfected A2780 cells treated with $50 \mu \mathrm{M}$ cisplatin. Data are presented as mean value from four independent experiments presented as fold change of cisplatin treatment. Error bars represent SEM.

up-regulation of RBM3 upon cisplatin-induced stress, however, it would still be of interest to investigate the potential role of RBM3 in DNA repair response given the observed decrease of RBM3-silenced cells arrested in $\mathrm{G} 2 / \mathrm{M}$ upon cisplatin treatment.

Apart from our previous study in breast cancer [16], there are to our knowledge no other published data on the prognostic impact of tumor-specific RBM3 expression in human cancer. However, RBM3 has been identified as one of five down-regulated genes in an in vitro model of melanoma progression [34], implying a beneficial impact on prognosis also in this cancer form. Contrasting in vitro data have also been published, whereby RBM3 has been proposed to be a proto-oncogene stabilizing COX-2 mRNA levels and protecting against mitotic catastrophe in colorectal cancer cell lines [27].

A common observation in human tissue is that RBM3 is up-regulated in cancer $[27,28]$ and in proliferating non-malignant cells [28], compared to normal cells. Quite in line with these findings, we found that siRNA mediated knockdown of RBM3 resulted in reduced proliferation in A2780 cells, which might in part explain the reduced cisplatin sensitivity and also the relatively modest reduction in apoptosis upon treatment in siRBM3 transfected A2780 cells. Yet, as a consequence of the observation that RBM3 seems to be necessary for the maintenance of cellular integrity during various stress conditions, it has been hypothesized that targeting RBM3 could prove to be an efficient novel therapeutic strategy against cancer $[27,35]$. This viewpoint is challenged by the results presented here, but, evidently, drug induced effects by RBM3 modulation seem to differ between different cell line models, as down-regulation of RBM3 has been associated with enhanced response to adriamycin and cisplatin in androgen dependent but not androgen-independent prostate cancer cells [35]. To what extent this variation is true in human tumors 
remains to be elucidated but to our knowledge, in contrast to ovarian cancer, prostate cancer is not routinely treated with platinum-based chemotherapeutic agents. It should also be emphasized that, in a translational context, the proposal that RBM3 is a proto-oncogene activated in response to adverse cellular conditions [27] does not contradict the findings that its presence in an established tumor is associated with a favourable patient outcome as such findings would not have taken patient treatment into account.

The coincidence of a beneficial prognostic impact of RBM3 expression in EOC both at the mRNA and protein level demonstrated here is particularly relevant from a translational perspective as it would justify using IHC, which is a simpler, faster and less costly method than RT-PCR in the clinical setting. In our previous study in breast cancer [16], the favourable prognostic impact of RBM3 was assessed by IHC in two independent patients cohorts using a polyclonal, monospecific antibody, initially developed within the HPA programme $[23,36]$. In the present study, the favourable prognostic impact of RBM3 expression in EOC was demonstrated at both the gene expression and protein levels in two relatively large independent cohorts. RBM3 protein expression was assessed using a monoclonal antibody which displayed a single band of the expected size on Western blot and further validation showed a decreased RBM3 expression in siRNA transfected A2780 cells compared to controls, both as assessed by IHC and Western blotting. Notably, the previously used polyclonal antibody has also been validated in the A2780 cells with similar results to the monoclonal antibody; e.g. differential expression in A2780 and A2780-Cp70 cells and decreased expression in siRNA transfected cells (data not shown). Furthermore, analysis of the tumor specimens in Cohort II using the antibody that was used in the breast cancer study [16] yielded concordant results regarding the prognostic impact of tumor-specific RBM3 expression (data not shown).

In breast cancer, nuclear RBM3 expression was associated with favourable clinicopathological parameters, including hormone receptor status [16]. In this study, we found no association between RBM3 and ER or PR expression in EOC as assessed by IHC. This observation indicates that RBM3 might have different functions in the context of estrogen-related signalling in breast cancer and ovarian cancer. The potential clinical relevance of this is however less evident as the beneficial effect of high RBM3 expression in breast cancer was independent of tamoxifen treatment.

\section{Conclusions}

Here, we present data from two independent patient cohorts demonstrating that expression of the RNA- binding protein RBM3, both at the mRNA and protein levels, is associated with a good prognosis in epithelial ovarian cancer. Furthermore, we show that decreased RBM3 expression confers reduced platinum sensitivity in ovarian cancer cells. These findings indicate that RBM3 may be a useful prognostic and treatment predictive marker in epithelial ovarian cancer.

\section{Additional material}

\begin{abstract}
Additional file 1: Expression of the apoptosis regulating proteins Bcl-2 and Bax in A2780 and A2780-Cp70 cells and siRBM3 transfected $\mathbf{A} \mathbf{2 7 8 0}$ cells compared to controls. Western blot analysis of (A) Bcl2 expression in A2780, A2780-Cp70 and siRBM3 transfected A2780 cells and Bax expression in (B) A2780 and A2780-Cp70 cells and (C) siRBM3 transfected A2780 cells.

Additional file 2: Cisplatin treatment does not affect the protein level of RBM3 or the siRNA-mediated down-regulation of RBM3. (A) Protein expression of RBM3 was examined by immunoblotting in A2780 cells treated with various concentrations of cisplatin for $1 \mathrm{~h}$ followed by 48 hrs culture in fresh drug-free media. (B) siRBM3 transfected A2780 cells were, 24 hrs post-transfection, treated with $50 \mu \mathrm{M}$ cisplatin for $1 \mathrm{~h}$ followed by $48 \mathrm{hrs}$ culture in fresh drug-free media whereby RBM3 remained down-regulated as shown by immunoblotting.

Additional file 3: The effects of RBM3 down-regulation on cell viability, cell cycle characteristics and apoptosis in A2780 cells. (A) Cell viability was evaluated by WST-1 assay in siRBM3 transfected A2780 cells. Data are presented as mean values from five independent experiments performed in triplicates presented as percentage of viable cells relative to si-control transfected cells. Error bars represent SEM. (B) Cell cycle phase distribution and $(\mathbf{C})$ fraction of apoptotic cells were analysed by flow cytometry in siRBM3 transfected A2780 cells. Data are presented as mean value from four independent experiments. Error bars represent SEM.
\end{abstract}

\section{Acknowledgements}

We thank Prof Robert Brown for kindly providing the A2780 and A2780Cp70 cells.

This study was supported by grants from the Knut and Alice Wallenberg Foundation, the Swedish Cancer Society, Gunnar Nilsson's Cancer Foundation, the Crafoord Foundation, and the Research Funds of Malmö University Hospital. The UCD Conway Institute is funded by the Programme for Third Level Institutions (PRTLI), as administered by the Higher Education Authority (HEA) of Ireland.

We thank Elise Nilsson for excellent technical assistance.

\section{Author details}

${ }^{1}$ Center for Molecular Pathology, Department of Laboratory Medicine, Lund University, Skåne University Hospital, Malmö, Sweden. ${ }^{2}$ UCD School of Biomolecular and Biomedical Science, UCD Conway Institute, University College Dublin, Belfield, Dublin 4, Ireland. '3Division of Oncology, Department of Clinical Sciences, Lund University, Skåne University Hospital, Lund, Sweden. ${ }^{4}$ School of Medicine and Medical Science, Conway Institute, University College Dublin, Dublin, Ireland. ${ }^{5}$ Division of Surgery, Department of Clinical Sciences, Lund University, Skåne University Hospital, Malmö, Sweden. ${ }^{6}$ The Malmö Diet and Cancer Study, Skåne University Hospital, Malmö, Sweden. ${ }^{7}$ Department of Biotechnology, AlbaNova University Center, Royal Institute of Technology, Stockholm, Sweden. ${ }^{8}$ Department of Genetics and Pathology, Rudbeck Laboratory, Uppsala University, Uppsala, Sweden.

\section{Authors' contributions}

Å participated in the data collection, performed the statistical analysis, carried out the functional studies and drafted the manuscript. DB participated in the data collection, performed statistical analysis, and helped to draft the manuscript. BN constructed the TMAs and participated in the 
data collection. DPO participated in the data collection. JE participated in the design of the study and helped draft the manuscript. MAK assisted with the data collection and helped draft the manuscript. IBJ assisted with the statistical analysis. JM assisted with data collection and helped to draft the manuscript. JB assisted with collection of clinical data. MU assisted with data collection and participated in its design. FP assisted with data collection and helped to draft the manuscript. KJ conceived of the study, participated in its design and coordination and helped to draft the manuscript. All authors read and approved the final manuscript.

\section{Competing interests}

The authors declare that they have no competing interests.

Received: 14 April 2010 Accepted: 20 August 2010

Published: 20 August 2010

\section{References}

1. Jemal A, Siegel R, Ward E, Hao Y, Xu J, Thun MJ: Cancer statistics, 2009. CA Cancer J Clin 2009, 59:225-249.

2. Kelland L: The resurgence of platinum-based cancer chemotherapy. Nat Rev Cancer 2007, 7:573-584.

3. Han ES, Lin P, Wakabayashi M: Current status on biologic therapies in the treatment of epithelial ovarian cancer. Curr Treat Options Oncol 2009, 10:54-66.

4. Tummala MK, McGuire WP: Recurrent ovarian cancer. Clin Adv Hematol Oncol 2005, 3:723-736.

5. Siddik ZH: Cisplatin: mode of cytotoxic action and molecular basis of resistance. Oncogene 2003, 22:7265-7279.

6. Jamieson ER, Lippard SJ: Structure, Recognition, and Processing of Cisplatin-DNA Adducts. Chem Rev 1999, 99:2467-2498.

7. Wang D, Lippard SJ: Cellular processing of platinum anticancer drugs. Nat Rev Drug Discov 2005, 4:307-320.

8. Wright CF, Oswald BW, Dellis S: Vaccinia virus late transcription is activated in vitro by cellular heterogeneous nuclear ribonucleoproteins J Biol Chem 2001, 276:40680-40686.

9. Burd CG, Dreyfuss G: Conserved structures and diversity of functions of RNA-binding proteins. Science 1994, 265:615-621.

10. Sutherland LC, Rintala-Maki ND, White RD, Morin CD: RNA binding motif (RBM) proteins: a novel family of apoptosis modulators? J Cell Biochem 2005, 94:5-24

11. Kita H, Carmichael J, Swartz J, Muro S, Wyttenbach A, Matsubara K, Rubinsztein DC, Kato K: Modulation of polyglutamine-induced cell death by genes identified by expression profiling. Hum Mol Genet 2002, 11:2279-2287.

12. Derry JM, Kerns JA, Francke U: RBM3, a novel human gene in Xp11.23 with a putative RNA-binding domain. Hum Mol Genet 1995, 4:2307-2311.

13. Danno S, Nishiyama H, Higashitsuji H, Yokoi H, Xue JH, Itoh K, Matsuda T, Fujita J: Increased transcript level of RBM3, a member of the glycine-rich RNA-binding protein family, in human cells in response to cold stress. Biochem Biophys Res Commun 1997, 236:804-807.

14. Ponten $F$, Jirstrom $K$, Uhlen M: The Human Protein Atlas-a tool for pathology. J Pathol 2008, 216:387-393.

15. Bjorling E, Lindskog C, Oksvold P, Linne J, Kampf C, Hober S, Uhlen M, Ponten F: A web-based tool for in silico biomarker discovery based on tissue-specific protein profiles in normal and cancer tissues. Mol Cell Proteomics 2008, 7:825-844.

16. Jogi A, Brennan DJ, Ryden L, Magnusson K, Ferno M, Stal O, Borgquist S, Uhlen M, Landberg G, Pahlman S, et al: Nuclear expression of the RNAbinding protein RBM3 is associated with an improved clinical outcome in breast cancer. Mod Pathol 2009, 22:1564-1574.

17. Tothill RW, Tinker AV, George J, Brown R, Fox SB, Lade S, Johnson DS, Trivett MK, Etemadmoghadam D, Locandro B, et al: Novel molecular subtypes of serous and endometrioid ovarian cancer linked to clinical outcome. Clin Cancer Res 2008, 14:5198-5208.

18. Bolstad BM, Irizarry RA, Astrand M, Speed TP: A comparison of normalization methods for high density oligonucleotide array data based on variance and bias. Bioinformatics 2003, 19:185-193.

19. Moody SE, Perez D, Pan TC, Sarkisian CJ, Portocarrero CP, Sterner CJ, Notorfrancesco KL, Cardiff RD, Chodosh LA: The transcriptional repressor Snail promotes mammary tumor recurrence. Cancer Cell 2005, 8:197-209.
20. Berglund G, Elmstahl S, Janzon L, Larsson SA: The Malmo Diet and Cancer Study. Design and feasibility. J Intern Med 1993, 233:45-51.

21. Berglund G, Eriksson KF, Israelsson B, Kjellstrom T, Lindgarde F, Mattiasson I, Nilsson JA, Stavenow L: Cardiovascular risk groups and mortality in an urban swedish male population: the Malmo Preventive Project. J Intern Med 1996, 239:489-497.

22. Kononen J, Bubendorf $L$, Kallioniemi A, Barlund M, Schraml P, Leighton S, Torhorst J, Mihatsch MJ, Sauter G, Kallioniemi OP: Tissue microarrays for high-throughput molecular profiling of tumor specimens. Nat Med 1998, 4:844-847.

23. Nilsson P, Paavilainen L, Larsson $K$, Odling J, Sundberg M, Andersson AC, Kampf C, Persson A, Al-Khalili Szigyarto C, Ottosson J, et al: Towards a human proteome atlas: high-throughput generation of mono-specific antibodies for tissue profiling. Proteomics 2005, 5:4327-4337.

24. Berglund L, Bjorling E, Jonasson K, Rockberg J, Fagerberg L, Al-Khalili Szigyarto C, Sivertsson A, Uhlen M: A whole-genome bioinformatics approach to selection of antigens for systematic antibody generation. Proteomics 2008, 8:2832-2839.

25. Lofstedt T, Jogi A, Sigvardsson M, Gradin K, Poellinger L, Pahlman S, Axelson $\mathrm{H}$ : Induction of ID2 expression by hypoxia-inducible factor-1: a role in dedifferentiation of hypoxic neuroblastoma cells. J Biol Chem 2004, 279:39223-39231.

26. Wellmann S, Buhrer C, Moderegger E, Zelmer A, Kirschner R, Koehne P, Fujita J, Seeger K: Oxygen-regulated expression of the RNA-binding proteins RBM3 and CIRP by a HIF-1-independent mechanism. J Cell Sci 2004, 117:1785-1794

27. Sureban SM, Ramalingam S, Natarajan G, May R, Subramaniam D, Bishnupuri KS, Morrison AR, Dieckgraefe BK, Brackett DJ, Postier RG, et al: Translation regulatory factor RBM3 is a proto-oncogene that prevents mitotic catastrophe. Oncogene 2008, 27:4544-4556.

28. Wellmann S, Truss M, Bruder E, Tornillo L, Zelmer A, Seeger K, Buhrer C: The RNA-binding protein RBM3 is required for cell proliferation and protects against serum deprivation-induced cell death. Pediatr Res 2010, 67:35-41.

29. Martinez-Arribas F, Agudo D, Pollan M, Gomez-Esquer F, Diaz-Gil G, Lucas R, Schneider J: Positive correlation between the expression of $\mathrm{X}$ chromosome RBM genes (RBMX, RBM3, RBM10) and the proapoptotic Bax gene in human breast cancer. J Cell Biochem 2006, 97:1275-1282.

30. Kolfschoten GM, Hulscher TM, Schrier SM, van Houten VM, Pinedo HM, Boven E: Time-dependent changes in factors involved in the apoptotic process in human ovarian cancer cells as a response to cisplatin. Gynecol Oncol 2002, 84:404-412.

31. Danno S, Itoh K, Matsuda T, Fujita J: Decreased expression of mouse Rbm3, a cold-shock protein, in Sertoli cells of cryptorchid testis. Am J Pathol 2000, 156:1685-1692.

32. Williams DR, Epperson LE, Li W, Hughes MA, Taylor R, Rogers J, Martin SL, Cossins AR, Gracey AY: Seasonally hibernating phenotype assessed through transcript screening. Physiol Genomics 2005, 24:13-22.

33. Lebsack TW, Fa V, Woods CC, Gruener R, Manziello AM, Pecaut MJ, Gridley DS, Stodieck LS, Ferguson VL, Deluca D: Microarray analysis of spaceflown murine thymus tissue reveals changes in gene expression regulating stress and glucocorticoid receptors. J Cell Biochem 2010, 110(2):372-81.

34. Baldi A, Battista T, De Luca A, Santini D, Rossiello L, Baldi F, Natali PG, Lombardi D, Picardo M, Felsani A, Paggi MG: Identification of genes downregulated during melanoma progression: a cDNA array study. Exp Dermatol 2003, 12:213-218.

35. Zeng $Y$, Kulkarni P, Inoue T, Getzenberg RH: Down-regulating cold shock protein genes impairs cancer cell survival and enhances chemosensitivity. J Cell Biochem 2009, 107:179-188.

36. Uhlen M, Bjorling E, Agaton C, Szigyarto CA, Amini B, Andersen E, Andersson AC, Angelidou P, Asplund A, Asplund C, et al: A human protein atlas for normal and cancer tissues based on antibody proteomics. $\mathrm{Mol}$ Cell Proteomics 2005, 4:1920-1932.

doi:10.1186/1479-5876-8-78

Cite this article as: Ehlén et al:: Expression of the RNA-binding protein RBM3 is associated with a favourable prognosis and cisplatin sensitivity in epithelial ovarian cancer. Journal of Translational Medicine 2010 8:78. 